\title{
Resonancias históricas y biográficas a la constitución del estudiante como actor político
}

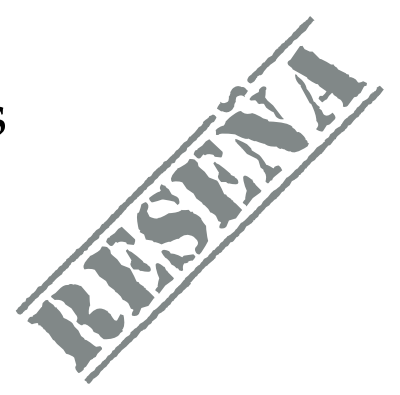

GuAdalupe Olivier TÉllez

Secretaria académica de la Universidad Pedagógica Nacional. Tiene estudios en sociología y de doctorado en pedagogía, ha sido Secretaria General del Consejo Mexicano de Investigación Educativa A. C. (COMIE), y activa colaboradora de la Red Mexicana de Estudios de los Movimientos Sociales.

Los movimientos estudiantiles son actores protagónicos dentro de los movimientos sociales que han marcado los siglos XX y XXI. No solo definen hitos históricos, sino también resonancias biográficas de sus militantes cuya acción colectiva trasciende en el tiempo. El libro Cien años del Movimiento Estudiantil, coordinado por Imanol Ordorika, Roberto Rodríguez-Gómez y Manuel Gil Antón, ejemplifica con casos emblemáticos, cómo los estudiantes de contextos y tiempos distintos se constituyen como piezas clave en la transformación de la historia reciente: con demandas educativas o sin ellas, vinculándose a causas sociales de diverso signo, tomando las calles, trasgredien-

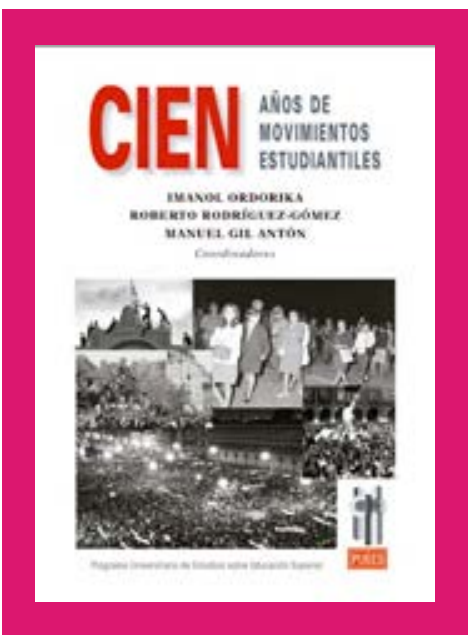
do el espacio público, imponiendo el pensamiento de su generación muchas veces de manera espontánea, y siempre o casi siempre, haciendo suyo el momento contencioso.

Es un texto emanado del Seminario de Educación Superior de la UNAM, que reúne un entramado de estudios académicos y análisis testimonial de primera mano. Es precisamente en esta articulación como se devela, en su conjunto, una historia crítica sobre los procesos de confrontación, los ciclos y repertorios de protesta, las implicaciones políticas y sociales, la posibilidad de cada movimiento de conformar o no un activista permanente (Fillieule y Neveu, 2019) vinculado a la arena política de su tiempo, que de manera ineludible, definieron trayectorias de vida que a la larga, dieron lugar a una cultura política constituida y a la vez constituyente de nuevos procesos que han marcado cambios fundamentales a lo largo de cien años.

Esta articulación se muestra en el libro a través de cuatro apartados organizados de manera cronológica: 1) Movimientos autonomistas en América Latina; 2) Movimientos estudiantiles de los sesenta; 3) Movimientos por la democracia y ajuste estructural de los ochenta y principios de los 
noventa; y 4) Movimientos del nuevo siglo. Internamente, cada uno de estos apartados busca integrar trabajos de corte académico con otros de tipo analítico-testimonial mostrándose en lo general algunos de los movimientos estudiantiles sucedidos en México, Argentina, Colombia, Chile, Perú, Cuba, Estados Unidos y Francia. Los 16 capítulos que componen el total del libro aportan no solo al campo de estudio de los movimientos sociales, sino de la propia historia social contemporánea. Es verdad que en sus líneas no se agota la totalidad de los movimientos estudiantiles que se presentaron entre los siglos XX y XXI, incluso los coordinadores del texto reconocen que no es un trabajo que pretenda ser exhaustivo.

En términos regionales se extrañan, por ejemplo, los movimientos en Bolivia, Brasil, Ecuador y Venezuela (Meyer, 2008); en México, a propósito, se recuerda la enorme fuerza de los movimientos de estudiantes normalistas desde la segunda mitad del siglo Xx, incluyendo desde luego el movimiento por los Desaparecidos de Ayotzinapa (Ramírez, 2018). Y también un importante movimiento estudiantil en los años recientes, el del Instituto Politécnico Nacional en 2014 "Todos somos Politécnico". Y en tal sentido, en una reflexión futura, valdría la pena incorporar el movimiento estudiantil feminista.

La primera parte, "Movimientos autonomistas en América Latina", cuenta con tres trabajos. En el primero, el trabajo de Diego Tatián, se plantea lo que podríamos llamar la madre de los movimientos estudiantiles del siglo XX: la insurrección estudiantil de Córdoba en 1918. A partir de un conocimiento histórico profundo del contexto y del relato detallado del movimiento, se desarrolla así un ensayo crítico a partir de la reconstrucción histórico-política de la Universidad de Córdoba y de la configuración del contexto ideológico cultural de esta región argentina.

El planteamiento del autor sobre el abordaje desde una arqueología política se convierte en la piedra angular del texto. Una de las dimensiones que se destacan de ello, es la relativa a las contradicciones que considero son las que más destacan del capítulo. Una de ellas es la que se encuentra en el seno de la sociedad cordobense, cuya expresión se muestra en la cultura conservadora de la Universidad de Córdoba considerada de fuerte influencia clerical, frente al corazón revolucionario de la Reforma asentada en el Manifiesto Liminar de la Reforma Universitaria de 1918 con un posicionamiento básicamente anticapitalista y latinoamericanista.

Este movimiento estudiantil, como en otros contextos, se vinculó fuertemente con el movimiento obrero cuyo antecedente fue la creación de universidades populares y clases para obreros desde 1916. La articulación con estos procesos, dotaron relevantemente de contenido político al movimiento universitario. Es necesario señalar que la noción de autonomía en realidad fue un aspecto que se incorporó paulatinamente, no fue un planteamiento que naciera con el movimiento en su fase inicial (Tatián, 2019, p.25).

En este mismo tema, Rodríguez-Gómez desde un trabajo de corte académico, plantea el sustento teórico del enfoque autonomista que ha privado en las universidades latinoamericanas. Lo aborda en dos partes: en la primera, debate acerca de lo que se ha dicho sobre la autonomía. Plantea una suerte de estado del arte ubicándose, en el análisis de la influencia del Movimiento de Córdoba, en otros movimientos estudiantiles que propug- 
naban por la autonomía, y enfatizando la importancia de ubicar las particularidades contextuales. En la segunda parte, aborda tres casos de movimiento reformista: Argentina, Perú y Cuba. En los tres casos se muestra una articulación política entre las protestas y procesos institucionales o meramente estudiantiles y de los movimientos de oposición a los regímenes políticos de cada país. La autonomía se convierte, en todo caso, en una noción estructurante entre ambos ámbitos de lucha de los estudiantes.

El autor reconoce grosso modo dos posturas en la literatura sobre la Reforma de Córdoba, una, que le atribuye a esta una condición sine qua non para la réplica que se produjo en las universidades latinoamericanas en los años posteriores a 1918. Y la otra es la que atribuye, más bien, la convergencia de aspectos socioeconómicos y políticos de la región latinoamericana que produce un escenario para los movimientos de autonomía universitaria y en adición a las particularidades propias de las instituciones.

La hipótesis del autor es una adición de ambas posturas, pues reconoce que el ideario de Córdoba influyó irremediablemente en los movimientos autonomistas, pero las condiciones contextuales y coyunturales determinaron la orientación del movimiento, el tipo de agenda que se planteó, los resultados que se obtuvieron y sus consecuencias posteriores (Rodríguez-Gómez, 2019, p.48). En síntesis, no podría entenderse la autonomía universitaria en la región latinoamericana sin la movilización estudiantil, ya sea como protagonista o como impulsor directo del proceso.

Se concluye esta primera parte con el caso de México abordado por Renate Marsiske. Es un trabajo académico que se ubica, en primera instancia, en la definición de autonomía. Así, hace un recorte temporal para analizar las implicaciones del término en el contexto de la lucha estudiantil en México, lo remonta a los antecedentes no solo de la Universidad de Córdoba, sino a procesos de más larga data pues ubica las primeras expresiones de autonomía en México, desde finales del siglo XIX y su resignificación en 1929.

Asimismo, muestra un marco conceptual que ubica diferentes perspectivas de autonomía universitaria. La autonomía en tal caso, la plantea como una idea contingente que se construye y también determina su funcionamiento respondiendo a los procesos de la época por la que transita la universidad. La autonomía tiene un sentido político. Esto explica, por ejemplo, los distintos momentos en los que la universidad se encuentra amenazada en su autonomía frente a distintos procesos gubernamentales, cuyas políticas acercan en menor o mayor medida la intervención o regulación estatal (Marsiske, 2019, p.63).

En realidad, los proyectos de autonomía universitaria en México abundaron desde 1917, pero el contexto de los gobiernos posrevolucionarios no veía con buenos ojos que la institución universitaria se saliera de su control porque se pensaba en un proyecto de unidad nacional a través de la educación, donde la universidad fuera el instrumento de acercamiento con las clases populares. Este es un aspecto muy importante, pues a diferencia de otras universidades latinoamericanas donde sus estudiantes luchaban contra gobiernos dictatoriales, en México se venía de una Revolución donde uno de sus pilares estaba en contra de la perspectiva y dominio clerical en la educación, lo cual plantea un escenario muy distinto a otros casos latinoamericanos. 
El movimiento estudiantil en México planteó, centralmente en sus consignas, una postura antiimperialista y anticlerical, amén de la reformulación del plan de estudios, el sistema de examinaciones y un conjunto de demandas respecto a la duración de los ciclos escolares del bachillerato, entre otros que se consiguieron tras el movimiento. Sin embargo, el punto que está en el trasfondo del logro de la autonomía es la recomendación de Manuel Puig Casauranc al presidente Emilio Portes Gil para que se concediera la autonomía, y con ello adquirir la responsabilidad y la posibilidad de resolver, por sí misma, sus problemas. Fue prácticamente deshacerse de la Universidad (Marsiske, 2019, p.85), y el 10 de julio de 1929 se promulga la Ley Orgánica de la Universidad Nacional Autónoma de México después de 68 días de huelga.

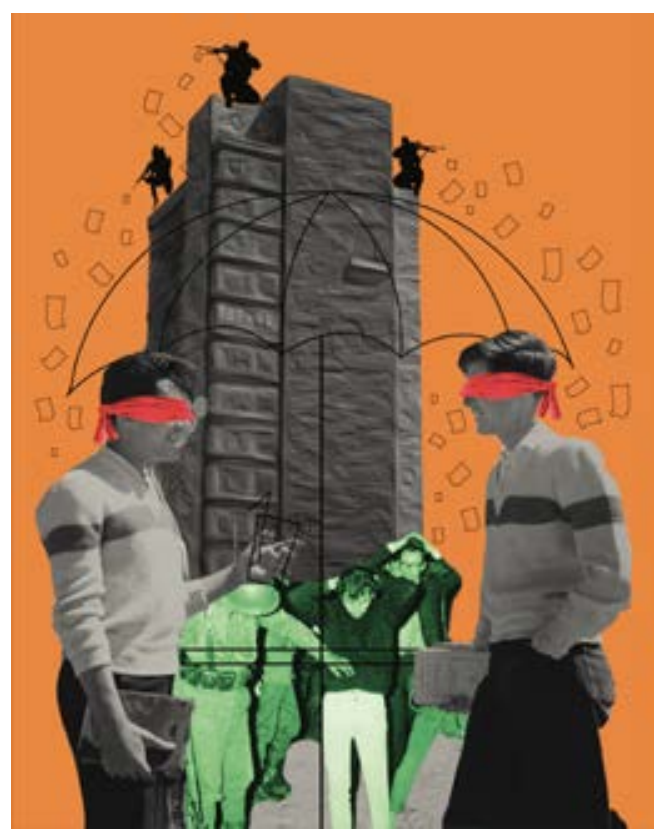

En la segunda parte del libro, "Revoluciones estudiantiles de los sesenta", cuenta con cuatro trabajos. Se inicia con un extraordinario relato de Todd Gitlin, quien desde su experiencia como militante ubica el contexto de los movimientos estudiantiles de los sesenta en Estados Unidos. La segregación racial, la protesta antimilitarista, la resistencia del movimiento de los anti-anticomunistas son tan solo algunos de los aspectos que cruzaban por las universidades en aquel momento, aunque con una respuesta minoritaria. Pero, sin duda, el mayor detonante fue contra la Guerra en Vietnam. En palabras del autor, la Guerra de Vietnam fue la "generalización del espíritu del movimiento estudiantil" (Gitlin, 2019, p.105).

En 1969 pasó de ser un movimiento solamente estudiantil a otro que conjuntó más sectores, incluso integró protestas de soldados, ministros religiosos y otras organizaciones civiles. Fue una época creciente de manifestaciones que frenaron de algún modo la guerra nuclear, aunque no todos aquellos que se movilizaron eran revolucionarios. A pesar de ello hubo una escalada de romanticismo de izquierda que soñaba con un escenario revolucionario, entre ellos estaban divididos los maoístas y hochiminhistas.

Atrás de la organización Estudiantes por una Sociedad Democrática (SDS, por sus siglas en inglés), estaba una perspectiva de lo que denominaron nueva izquierda que no solo estaba contra cualquier vestigio de autoridad institucional, sino también contra cualquier forma jerárquica dentro del movimiento. Este aspecto recuerda los movimientos estudiantiles más recientes, que justamente plantean esta forma no vertical de organización. 
La SDS estaba apoyada por algunas organizaciones como las Panteras Negras, quienes admiraban la Revolución Cubana y las organizaciones estudiantiles cada vez se radicalizaban más, colocando explosivos en distintos lugares simbólicos, lo que alertó al gobierno especialmente y, por supuesto, lo consideraron como de alta gravedad.

No fue un movimiento estudiantil en el sentido de que no había demandas estudiantiles propiamente, este es un ejemplo de la participación estudiantil como organización imbricada en protestas sociopolíticas. A partir de los setenta el movimiento estudiantil dejó de tener la misma fuerza que en la década pasada. Entraron a relevarlo varios movimientos: el feminista, el ambientalista, el antibelicista y el que apoyaba la homosexualidad, por ejemplo. Una característica de los movimientos sociales en esta época en Estados Unidos es que, por lo regular, se pretendía una dirección democrática, pero no revolucionaria.

En ese mismo tenor, el capítulo de Obéy Ament sobre el movimiento de Mayo del 68 en Francia, da nuevamente el ejemplo de la articulación entre el movimiento estudiantil con el obrero. Mismo que se considera el movimiento más grande en la historia de ese país europeo. La clave para entenderlo fueron las grandes movilizaciones sociales a nivel internacional que influyeron en los estudiantes franceses.

La autora reconoce cuatro aspectos contextuales que considera relevantes: 1) el auge de los Movimientos de Liberación Nacional, específicamente el de Argelia; 2) la Guerra de Vietnam y la Revolución Cubana, incluido el asesinado del Ché Guevara en Bolivia, en 1967; 3) la Guerra Fría y la crisis del estalinismo; 4) los cambios socioeconómicos en los países imperialistas que produjeron movimientos estudiantiles en Alemania, Estados Unidos y Japón, producto, según su análisis, del cambio de la composición del estudiantado, gracias a la expansión de la educación superior, pues los estudiantes no eran ya solamente de las élites sino también de sectores populares y las clases medias.

En marzo de 1968 entró la policía a la Universidad de Nanterre, con ello comenzó una creciente oleada de movilizaciones. En el mayo francés se encontraba como presidente Charles de Gaulle. Representaba el poder conservador, justo como era la sociedad francesa de entonces. Ordenó la represión física contra los estudiantes, asunto poco usual en Francia. Un dato relevante, además de que todos eran hombres, el feminismo aún era muy incipiente (Abel, 2019, p.124), es que igual que en Estados Unidos, desde la protesta del 20 de marzo, no se distinguió un líder, ni organización preestablecida, esto no quiere decir que no hubiera un núcleo que reunía y organizaba actividades.

Un hecho que queda como ícono en la historia fue la movilización contra la represión, que radicaliza el movimiento popular. Los adoquines son arrancados del piso como barricadas, tal como en la Revolución francesa y en la Comuna de París. Se revela con ello la importancia de la memoria colectiva y las resonancias históricas, pero también las biográficas en cada uno de los militantes (Olivier y Tamayo, 2019 y 2017), justo como este libro aportará para las actuales generaciones y las venideras.

París paralizado, el país entero, sin vuelos, ni trasportes, ni labores en las fábricas, ni en las tiendas, ni en ningún lado. A finales de mayo se dis- 
tinguía un vacío de poder y, por lo tanto, una total crisis de legitimización de De Gaulle. La derecha constata que el movimiento no tenía una salida política y de ello se aprovecha el presidente. También los partidos socialista y comunista, quienes no tenían el control del movimiento, pretenden que este termine. No había una dirigencia, pero si conducción de sujetos un tanto anónimos que alentaron el movimiento. También estudiantes comunistas críticos que fungieron como ideólogos hasta cierto punto. Es destacable que el Partido Comunista Francés estaba aislado del movimiento.

A principios de junio la huelga terminó bajo una propuesta de negociación con los sindicatos rechazada por el sindicato estudiantil a quien no se le llamó. Los sindicatos y los partidos de izquierda contribuyeron a que finalizara la huelga. Se llamó a votaciones hacia finales de mes y ganó, con gran mayoría, la derecha. El movimiento confirmó los límites de la espontaneidad y la ausencia de una estrategia política de largo plazo. Una de las lecciones que queda para el presente es la importancia del apoyo popular y recuperación de la memoria histórica.

El tercer trabajo de este apartado es el del "Movimiento del 68 en México", documento escrito por Martínez della Roca a partir de una reflexión y análisis de su experiencia como dirigente del movimiento, plantea, en primer lugar, el contexto del modelo autoritario de gobierno en el país y su proceso de crisis. Es interesante su análisis sobre la sociedad mexicana de aquel momento pues la ubica como desarticulada, con sectores medios urbanos que dejaron de ser las bases de apoyo al gobierno como hasta entonces había sido. Señala que, desde el punto de vista sociológico y político el movimiento, derrotado a través de la utilización de las fuerzas militares, significó una acción represiva ejemplar del gobierno para recuperar y mostrar su fuerza al resto de la sociedad.

No obstante, desde el punto de vista histórico la resonancia social no tiene precedentes. Se constituye como un emblema, un bastión de lucha de las generaciones jóvenes y no tan jóvenes por más de cincuenta años en México. El pliego petitorio de entonces, aunque no fue cumplido en ninguna de sus demandas, sigue estando vigente. Cada año en sus conmemoraciones se renueva e incorporan otras demandas y sus fundamentos básicos han prevalecido en el tiempo.

Finalmente, un excelente trabajo de Zermeño cierra el segundo apartado. Desde la experiencia participativa y su solidez académica plantea un análisis del movimiento desde una retrospectiva que mira desde el presente. Plantea cuatro vertientes de los actores que se combinaron en el movimiento estudiantil, los denomina como: 1) Demócratas libertarios, aludiendo al paso de una represión inicial innecesaria a los estudiantes, a una demanda fundamental por las libertades; 2) Demócratas cultivados, asumiendo la ruptura cultural de la época en donde se gesta una contracultura que se confrontaba con los valores conservadores del México de antes de los sesenta, una nueva sociedad con la emergencia de nuevas formas de entender las relaciones sociales más diversas; 3) Demócratas reformistas, encarnado por las clases medias y la sorprendente actitud congruente del rector de la UNAM; 4) Demócratas revolucionarios, donde ubica a la corriente más radical de todas, los cuales fueron sectorizándose con el paso del tiempo, pero que consiguen la hegemonía de la dirigencia, son de 
hecho los principales interlocutores con el gobierno, por lo que se fortalecen, se radicalizan y se conectan con luchas sociales, obreras y campesinas que les dan una reorientación más amplia. Se fortalecen los grupos políticos, que emanan de la experiencia tanto de lucha como de formación en el contexto del movimiento, los sectores estudiantiles más politizados.

Es importante puntualizar respecto a las distinciones que hace el autor respecto a las movilizaciones similares en el mundo, en términos temporales, pues sugiere que el movimiento estudiantil mexicano de 1968 fue sustancialmente distinto, al menos en tres aspectos: en primer lugar, en que se convirtió en un movimiento político que perdió su médula que era la revolución cultural; en segundo lugar, se caracterizó por generar formas organizativas jerárquicas que se oponían a las tendencias de dirigencia democrática en el mundo, lo contradictorio es que se luchaba contra el autoritarismo; y por último, fue un movimiento que no contó con demandas educativas en su esencia (Zermeño, 2019, p.178). El punto de cohesión en todo caso fue el relativo a las libertades democráticas, pero en este enorme paraguas se cobijaron perspectivas y grupos muy disonantes. El análisis que establece sobre las limitaciones de la trascendencia de los movimientos sociales en México se los atribuye a la estructura piramidal autoritaria en la que seguimos inmersos. Por tales razones considera a los años setenta y ochenta como el triunfo del reformismo.

La tercera parte del texto, denominada "Por la democracia y contra el ajuste estructural", se inicia con el capítulo de Leticia Pogliaghi, quien dentro de una perspectiva académica plantea la participación de los estudiantes en la década de los ochenta, como ejes motores en la reconstrucción democrática en Argentina. De tal manera plantea el contexto de la última dictadura, las características del sistema universitario argentino y el escenario de la democracia. En realidad, el movimiento estudiantil de los setenta corresponde a la de la última dictadura militar, caracterizado por una fuerte intervención ideológico-política, represiva e intervencionista en las universidades, por ejemplo, la prohibición de actividades políticas en las universidades, la limitación de la libertad de cátedra, la violencia selectiva, entre otras. Esta época se caracterizó por la criminalización de la protesta, la intolerancia de la acción política ejecutada por grupos paramilitares, cesantías de docentes en labores educativas en universidades, desapariciones de estudiantes universitarios y secundarios.

En los ochenta, el contexto universitario era de poca fuerza política, pues gracias a las acciones de la dictadura quedó una matrícula reducida y profesores impuestos por el sistema. Sin embargo, se inició un periodo de apertura democrática que también se reflejó en las universidades. Se incluyeron políticas de gratuidad e ingreso abierto que ampliaron la matrícula e incre- 
mentó la planta docente como consecuencia. A pesar del fuerte control, represión e intervencionismo en las universidades en los setenta, una década después se recuperó una perspectiva democrática. También se recobraron fácilmente los procesos de politización. El restablecimiento de la autonomía universitaria fue lo que permitió procesos de unificación ideológica en los estudiantes y profesorado. En el caso de la universidad en Argentina, así como en los contextos que se han planteado con anterioridad, las universidades se constituyen como espacios esenciales de formación política y de vinculación con las luchas sociales y políticas fuera de las demandas meramente educativas.

De esta misma manera, Ament analiza el movimiento estudiantil en Francia en la década de los ochenta, a partir de un relato extraordinario sobre su participación como activista. Se centra en el conflicto de la Ley del 86, que polarizó dos posturas, por un lado, la educación universitaria para las élites y por otro la defensa de una Universidad de carácter igualitario. Contextualizando el caso, señala que desde los sesenta siempre estuvo latente en Francia el problema del ingreso a la universidad. Este tema se convirtió en un asunto medular en los ochenta, que a pesar de una idea un tanto filosófica sobre la igualdad, en los hechos, el acceso de las clases populares a la universidad era bastante más reducido que las clases medias y las élites.

En 1986, las movilizaciones también fueron de los sectores privilegiados, pues la Ley Savary implicaba la unificación de las instituciones públicas y privadas, estas últimas de corte religioso principalmente. La derecha reaccionó movilizando a un millón de personas. La buena lección de todo esto es considerar que la derecha también se mueve. En este sentido es importante recordar el contexto de la generalización e impulso de las privatizaciones o participación privada que se dio lugar en aquellos años. Se produjeron protestas crecientes y de nueva cuenta, como en el mayo 68, se adhirieron los sindicatos de trabajadores. En el fondo se encuentra una defensa a los principios de democracia y ejercicio de la ciudadanía para detener la fuerza de la derecha y sus políticas.

Por otro lado, el tercer trabajo de esta parte aborda el movimiento del Consejo Estudiantil Universitario entre 1986 y 1993, bajo el testimonio de Moreno, uno de los principales militantes. Para comprender cabalmente la esencia del movimiento es menester detenerse en el contexto de la crisis económica de los ochenta. Y en este sentido el autor revisa cómo se involucraron en el movimiento siendo estudiantes de nivel medio superior, específicamente del Colegio de Ciencias y Humanidades (CCH). Un aspecto importante es el antecedente y contexto del CCH, cómo surgió, quienes y cuál era el perfil del profesorado. Aquí vuelven a resurgir las resonancias históricas, biográficas y políticas, pues muchos de quienes integraban la planta docente habían 
sido participantes del movimiento de 1968. La estructura curricular del colegio heredaba una forma educativa innovadora que se oponía a los modelos del pasado que tanto se habían sido criticados en los sesenta.

Frente a ello, los estudiantes se enfrentaron al Plan Carpizo, que entre otras cosas planteaba el cese al paso automático a la universidad. Así, se establecieron brigadas en carreras y preparatorias no organizadas. Un aspecto destacable en el relato del autor es el de la participación de las mujeres en el movimiento. Reconoce la subordinación de las activistas a "tareas femeninas" y aunque había mucha representación de mujeres estas estaban al margen de los liderazgos principales o vocerías.

En esta misma temática se articula el texto de Imanol Ordorika, uno de los protagonistas más importantes del movimiento, que plantea una serie de fases que van desde el origen del conflicto, los antecedentes, los agravios, la herencia del movimiento, el desenlace y el legado. Esboza con mucha claridad los momentos del movimiento, su contexto, la vida cotidiana del quehacer político estudiantil y la valía de centrar la discusión en uno de los puntos que me parecen más relevantes hasta el día de hoy, las condiciones del ser estudiante. Es decir, un estudiante mexicano promedio en una institución pública, muy lejos del modelo ideal del universitario del primer mundo:

... Se trataba del ajuste estructural sobre la Universidad, la expresión manifiesta de la antidemocracia, el maltrato y desinterés sobre los estudiantes. Esos eran los agravios... (Ordorika, 2019, p.255)

De cierta forma, como en los movimientos estudiantiles posteriores, los participantes del movimiento se sentían los herederos del 68. Fueron influidos en las formas de organización, de representatividad y de acción política. Es importante resaltar que fueron precursores de la utilización de los medios masivos, en la medida que daba la época no solo en términos tecnológicos, sino también en las posibilidades restringidas de la apertura de los medios públicos y privados. Su estrategia de legitimidad se basó en los diálogos públicos. El balance del autor es que fue un movimiento exitoso en la medida que se ganaron demandas. Además, la organización representativa de estudiantes perduró de 1987 hasta 1992. Y, aun cuando se logró el cambio de Ley Orgánica, reconoce que es un movimiento que no siempre goza del reconocimiento de los movimientos estudiantiles.

La última parte del libro aborda los movimientos del siglo XXI. Inicia con el movimiento del Consejo General de Huelga 1999-2000, relato de la experiencia militante de Marcela Meneses Reyes. Desarrolla su versión del movimiento, a través de una vivencia que marca las esferas totales de la existencia de los participantes. Aborda uno de los aspectos más relevantes, aunque menos tratados en el conjunto de los estudios de los movimientos sociales: quienes eran estos estudiantes que participaron en 1999-2000, y sobre todo qué los llevó a participar en el movimiento. Puntualiza que usualmente se señalan a los héroes, a los protagonistas del movimiento, o bien a los críticos cuando se analiza el movimiento del CGH, pero un tema que considera medular y es el quiénes eran los que hacían la fuerza del movimiento en una escala distinta, en la vida cotidiana del movimiento. 
En el relato de Meneses la idea resonancia histórica y biográfica tiene un matiz distinto. Si para muchos movimientos existe un referente que hay que replicar, que inspira, que se considera continuar como legado, en otros casos el referente se da para superar formas y errores. Este es el caso que aquí se relata: no tener líderes, con una dirigencia colectiva para generar un movimiento horizontal. El referente del CGH no fueron más los movimientos estudiantiles del pasado, pero si había una inspiración y este era el EZLN, como su referente político y moral básico.

En 1999, el rector Francisco Barnés planteó la modificación del Reglamento General de Inscripciones y el Reglamento General de Exámenes. Esto produjo un fuerte detonante, y frente a la creciente movilización y la huelga de diez meses la configuración interna del movimiento sufrió polarizaciones importantes a lo largo de su largo proceso. Se produjeron fuertes confrontaciones a grado violento entre los dos fragmentos principales: los moderados y los ultras. El movimiento termina después de la intervención de la fuerza pública, de la represión y de más de 700 detenidos. Desde entonces se vislumbraban en México procesos de privatización en el sistema de educación pública, mientras que, en otros contextos, como en Chile, la puesta en marcha de políticas de mercado educativo estaban presentes desde años atrás.

El análisis del caso chileno lo presenta Marion Lloyd, quien plantea que el movimiento estudiantil en Chile tiene al menos 15 años. Es consecuencia de la orientación del modelo de educación implementada desde el pinochetismo y en un contexto de represión extrema, donde las universidades se convirtieron en el principal enemigo del sistema. La reducción de la matrícula, encarcelamientos y desapariciones fueron las estrategias para contener ideas y acciones de resistencia. El asunto de la politización de las universidades públicas y la incomodidad que esto generó para la implementación de políticas neoliberales más agresivas no es exclusivo de este país, de hecho lo vemos de manera muy clara y en una escalada cada vez mayor, conforme avanza el propio sistema y se impulsan al mismo tiempo, la incorporación de instituciones privadas y procesos de gestión de las instituciones públicas orientadas al modelo de mercado.

Chile es el pionero en Latinoamérica en la implementación de este modelo, lo cual a la larga generó enormes desigualdades en el acceso y permanencia 
en la educación superior, entre otras cosas, por los endeudamientos estudiantiles. En este sentido, la autora se centra en el movimiento que se generó dentro de los sucesos de 2011 y 2012 en la lucha por la gratuidad de la educación. El telón de fondo es el sistema de financiamiento de las universidades chilenas lo cual provocó en el gobierno de Sebastián Piñera, el gran paro nacional por la recuperación de la educación pública. El movimiento estudiantil chileno ha sido ejemplar para otros contextos y en diversos sentidos. Varios se destacan en el texto de Lloyd, por razones de espacio y siguiendo la lógica de la idea de resonancia, me centro solo en algunos aspectos. Ha sido un movimiento que en efecto ha durado en el tiempo por las formas creativas y no violentas de los activistas dentro de sus repertorios de movilización, lo cual ha logrado ampliar y sostener el movimiento gracias al apoyo de la sociedad.

Ha tenido figuras que han construido liderazgo y que, por lo tanto, han logrado ser, en los hechos, puntos de referencia clave, como en el caso de Camila Vallejo que además le imprimió un enfoque de género al movimiento. En su conjunto, la fuerza del movimiento ha logrado, quizá como ningún otro movimiento estudiantil, tener fuerte influencia sobre la política: “...primero, logró poner en crisis a Piñera, y después a Bachelet. Quizá ahora Piñera piensa, 'no me voy a exponer a una segunda derrota', por lo que está tomando muy en serio una nueva lógica en las políticas de educación superior en el país..." (Lloyd, 2019, p.303).

En una temporalidad similar, pero en Colombia, Juan Sebastián López Mejía aborda al movimiento estudiantil de entre los años 2010-2012. Plantea el contexto colombiano y su política educativa. Coincide así con los movimientos estudiantiles del siglo XXI en el tema de la gratuidad, recuperación de lo público, la no privatización por el impulso a la educación privada lucrativa y el enorme endeudamiento, como se ha explicado en el caso de Chile y como también veremos en adelante en el caso de los Estados Unidos.

Se plantea la problemática en relación con otras iniciativas, como la Ley del primer empleo, que en el fondo promovía mano de obra barata a las empresas, lo cual generó entre las otras situaciones señaladas arriba, el surgimiento en 2011 de la Mesa Amplia Nacional Estudiantil (MANE). El movimiento estudiantil colombiano como todos los que se han mostrado 
en esta reseña son mucho más complejos en su estructuración interna y en sus formas de lucha, baste decir, en este caso, que logra integrar a otros sectores, como, por ejemplo, una gran movilización del profesorado. Podría considerarse como un movimiento exitoso en tanto, gracias a la toma de Bogotá que reflejó la fuerza del movimiento, logra el retiro de la Ley de Educación Superior.

En esos años, también surge en México el Movimiento \#YoSoy132, pero en una situación absolutamente distinta, no tiene como fundamento ninguna demanda de carácter educativo, ni en alianza a otra organización o movimiento. Surge en el contexto electoral de las precampañas del año 2012 y de inicio motivada por estudiantes de universidades privadas. El trabajo de Karla Amozurrutia, participante del movimiento, muestra un relato desde su particular visión dentro del movimiento, lo cual enriquece el conjunto de estudios sobre este tema. Plantea el detonante del movimiento, el agravio por la descalificación al acto de rechazo al candidato del PRI, Enrique Peña Nieto y la derivación de las acciones mediáticas que darían lugar al movimiento \#YoSoy132.

Este movimiento fue creciendo inusitadamente y se le fueron agregando de manera muy importante, instituciones universitarias públicas. Fue un movimiento que se generalizó en el país y que además incorporó a estudiantes mexicanos que estudiaban en el extranjero. Rescata los principios organizativos claves a lo largo del siglo, que fue el carácter autónomo sin mediación de liderazgos o involucramiento de partidos y corrientes políticas. Ese fue uno de los puntos de erosión interna pues no pudo mantenerse como movimiento "puro", sobre todo cuando se integraron más fuerzas disímiles. Otro aspecto relevante es que al menos en los casos tratados en el texto como el chileno y colombiano, e incluso el del CGH, tenían un carácter antineoliberal, por lo menos en el sentido de la defensa a la educación pública y contra la privatización. Un punto de quiebre interno, importante en el \#YoSoy132, fue la integración de demandas que ya no eran compartidas en el momento inicial del movimiento, que era la democratización de los medios y de la vida política del país. Por ello es interesante contrastar los diversos estudios que se han realizado sobre este movimiento y contrastarlos con los testimonios de vida y comprender la complejidad inherente a estos procesos.

La cuarta parte del libro cierra con el trabajo de Alejandro González Ledesma y Héctor Vera, sobre los movimientos estudiantiles en Estados Unidos en el presente siglo. Es un trabajo de corte académico que muestra un balance y cifras detalladas sobre el papel de los estudiantes en el movimiento Occupy Wall Street, que más que un movimiento estudiantil en sí mismo más bien muestra de cómo el estudiantado universitario de instituciones disímeles se incorporó de manera muy vigorosa a un movimiento de la sociedad civil frente a las políticas capitalistas neoliberales. El problema de deuda educativa permitió que los estudiantes se incorporaran con mucha fuerza a un movimiento de más largo aliento y en consonancia internacional. Tampoco surgió de una consigna educativa propia, aunque sí se incorporó con mucha facilidad y fueron fuente de apoyo a la ocupación en la calle, vinculándose al núcleo del movimiento en la protesta contra las 
políticas neoliberales, es decir, se insertaron en la globalización la protesta antineoliberal capitalista.

De ahí se derivan lecciones, e importantes resonancias que los autores ubican en torno a la formación de una consciencia política y de la experiencia frente a la participación de uno de los movimientos recientes más importantes en un país desarrollado. Asuntos como el análisis de la deuda por los estudios universitarios, como en el caso chileno, el deterioro del trabajo académico o la posibilidad de creación de sindicatos universitarios, fueron legados posteriores que han incidido directamente en demandas de tipo educativo y que se plantearon como posibles acciones colectivas que fueron posteriores al movimiento.

Para cerrar el texto, Manuel Gil Antón plantea una serie de aspectos sobre el conjunto de los movimientos presentados en el texto, pero también a partir de los debates que se generaron en el seminario que da lugar al libro. Ubica la relevancia de la articulación entre los estudiosos de los movimientos estudiantiles con la incorporación de las voces de los protagonistas. Plantea en ello líneas para la reflexión y análisis que son producto tanto de los documentos publicados en el libro como las discusiones generadas en el seminario. De estas destaco las siguientes: 1) establecer la distinción entre movilizaciones y movimientos, que podría entenderse como la diferencia entre acciones colectivas y movimientos sociales; 2) ubicar la relevancia del contexto, en todo caso social y político; 3) ubicación del papel de lo histórico, el cual va ligado y da cobijo al punto anterior; 4) ubicación del peso decisivo de los cronistas; 5) la relación de los movimientos con otros actores sociales; 6) reflexión sobre los cambios generacionales y su impacto en la configuración de cada movimiento; 7) la heterogeneidad de los movimientos, pues no basta con que la etiqueta los ubique como estudiantiles, cuentan con una especificidad que los coloca en una particularidad que da lecciones distintas y complejas; y 8) la importancia de ubicar las formas comunicativas de los movimientos en su tiempo y espacio.

Para cerrar esta reseña, es importante decir que el libro muestra versiones del movimiento estudiantil de diverso signo, que ponen en la mira perspectivas que ubican al lector entre la historia oficial, los estudios académicos y el relato desde la vivencia. El lugar que ocuparon los participantes en los movimientos, así como el legado individual y colectivo es el eje de la memoria que revela su importancia a lo largo de las 400 páginas del texto, disponible, de manera gratuita, en la siguiente liga.

https://www.puees.unam.mx/publicaciones/Libros/OrdorikaEtAl2019_CienAniosDeMovimientosEstudiantiles.pdf 